\title{
TRANSFERENCE OF MAXIMAL MULTIPLIERS ON HARDY SPACES
}

\author{
DASHAN FAN AND ZHIJIAN WU
}

(Communicated by J. Marshall Ash)

\begin{abstract}
Based on the atomic decomposition of the Hardy space, we give a simple proof for a theorem of Liu and Lu (Studia Math. 105 (1993), 121-134), which discusses the relation between the maximal operators on $\mathbb{R}^{n}$ and on $\mathbb{T}^{n}$. More significantly, our proof shows that condition (1) in Liu and Lu's Theorem 1 is superfluous.
\end{abstract}

\section{INTRODUCTION}

Let $H^{p}\left(\mathbb{R}^{n}\right), 0<p<\infty$, be the Hardy spaces defined by [FS]

$$
H^{p}\left(\mathbb{R}^{n}\right)=\left\{f \in \mathscr{S}^{\prime}\left(\mathbb{R}^{n}\right),\left\|\Phi^{+} f\right\|_{L^{p}\left(\mathbb{R}^{n}\right)}<\infty\right\},
$$

where $\Phi^{+} f(x)=\sup _{t>0}\left|\Phi_{t} * f(x)\right|, \Phi_{t}(x)=t^{-n} \Phi(x / t)$, and $\Phi \in \mathscr{S}\left(\mathbb{R}^{n}\right)$ is a radial function satisfying $\int \Phi=1$. The corresponding periodic Hardy spaces are $H^{p}\left(\mathbb{T}^{n}\right)=\left\{f \in \mathscr{S}^{\prime}\left(\mathbb{T}^{n}\right),\left\|\tilde{\Phi}^{+} f\right\|_{L^{p}\left(\mathbb{T}^{n}\right)}<\infty\right\}$, where $\boldsymbol{\Phi}^{+} f(x)=$ $\sup _{t>0}\left|\tilde{\Phi}_{t} * f(x)\right|, \tilde{\Phi}_{t}(x)=\sum_{k \in \Lambda} \hat{\Phi}(t k) e^{2 \pi i k \cdot x}=C t^{-n} \sum_{k \in \Lambda} \Phi((x+k) / t)$ and $\Lambda$ is the unit lattice which is the additive group of points in $\mathbb{R}^{n}$ having integral coordinates.

Let $\lambda$ be a bounded continuous function on $\mathbb{R}^{n}$. For each $\varepsilon>0$, define

$$
\left(T_{\varepsilon} f\right)^{\wedge}(u)=\lambda(\varepsilon u) \hat{f}(u), \quad f \in L^{2}\left(\mathbb{R}^{n}\right) \cap H^{p}\left(\mathbb{R}^{n}\right),
$$

and

$$
\tilde{T}_{\varepsilon} f(x)=\sum_{k \in \Lambda} \lambda(\varepsilon k) a_{k}(f) e^{2 \pi i k \cdot x}, \quad f \in L^{2}\left(\mathbb{T}^{n}\right) \cap H^{p}\left(\mathbb{T}^{n}\right) .
$$

We say that $\lambda$ is a maximal multiplier on $H^{p}\left(\mathbb{R}^{n}\right)$ if $T^{*} f(x)=\sup _{\varepsilon>0}\left|T_{\varepsilon} f(x)\right|$ can be extended to a bounded operator from $H^{p}\left(\mathbb{R}^{n}\right)$ to $L^{p}\left(\mathbb{R}^{n}\right)$. Similarly, $\lambda$ is called a maximal multiplier on $H^{p}\left(\mathbb{T}^{n}\right)$ if $\widetilde{T}^{*} f(x)=\sup _{\varepsilon>0}\left|\widetilde{T}_{\varepsilon} f(x)\right|$ can be extended to a bounded operator from $H^{p}\left(\mathbb{T}^{n}\right)$ to $L^{p}\left(\mathbb{T}^{n}\right)$.

The relation between the maximal multipliers $T^{*}$ and $\widetilde{T}^{*}$ was first studied by Kenig and Tomas when $p>1$. Their result can be extended to the Lorentz

Received by the editors February 14, 1994 and, in revised form, April 4, 1994.

1991 Mathematics Subject Classification. Primary 42B30.

Key words and phrases. Maximal operator, Hardy spaces, atomic decomposition.

The first author was supported in part by a grant of The Graduate School Research Committee in University of Wisconsin-Milwaukee. 
space $L(p, q), p>1$ (see [F]). Recently, Liu and Lu [LL] studied the case of $0<p \leq 1$. Their main result is the following theorem.

Theorem 1 [LL]. Let $0<p \leq 1$, and let $\lambda$ be a bounded and continuous function on $\mathbb{R}^{n}$.

(i) Suppose that $\lambda$ is a maximal multiplier on $H^{p}\left(\mathbb{R}^{n}\right)$ such that

$$
\lim _{|x| \rightarrow \infty} \lambda(x)=\alpha
$$

exists. Then $\lambda$ is a maximal multiplier on $H^{p}\left(\mathbb{T}^{n}\right)$.

(ii) If $\lambda$ is a maximal multiplier on $H^{p}\left(\mathbb{T}^{n}\right)$, then $\lambda$ is a maximal multiplier on $H^{p}\left(\mathbb{R}^{n}\right)$.

Since the above condition (1) plays an important role in their proof, Liu and Lu asked (see p. 133 in [LL]) if condition (1) can be weakened any further.

In this note, we will show that condition (1) in Theorem 1 is superfluous. Based on the atomic decomposition of the Hardy space, our proof is much shorter and more direct than those in [LL]. The following is our main result.

Theorem 2. Let $\lambda$ be a continuous and bounded function on $\mathbb{R}^{n}, 0<p \leq 1$. If

$$
\left\|T^{*} f\right\|_{L^{p}\left(\mathbb{R}^{n}\right)} \leq C\|f\|_{H^{p}\left(\mathbb{R}^{n}\right)} \text { for all } f \in H^{p}\left(\mathbb{R}^{n}\right),
$$

then

$$
\left\|\tilde{T}^{*} \tilde{f}\right\|_{L^{p}\left(\mathbb{T}^{n}\right)} \leq C\|\tilde{f}\|_{H^{p}\left(\mathbb{T}^{n}\right)} \text { for all } \tilde{f} \in H^{p}\left(\mathbb{T}^{n}\right) .
$$

For the sake of simplicity, $C$ always denotes a positive constant which may vary at each of its occurrences.

To prove Theorem 2, we need to use the atomic characterization of the Hardy space. A regular $(p, 2, s)$ atom is a function $a(x)$ supported in some ball $B\left(x_{0}, \rho\right)$ satisfying

$$
\begin{gathered}
\|a\|_{2} \leq \rho^{-n / p+n / 2} ; \\
\int_{\mathbb{R}^{n}} a(x) P(x) d x=0
\end{gathered}
$$

for all polynomials $P(x)$ of degree less than or equal to $s$.

The space $H_{a}^{p, s}\left(\mathbb{R}^{n}\right), 0<p \leq 1$, is the space of all distributions $f \in \mathscr{S}^{\prime}\left(\mathbb{R}^{n}\right)$ having the form

$$
f=\sum c_{k} a_{k}
$$

and satisfying

$$
\sum\left|c_{k}\right|^{p}<\infty
$$

where each $a_{k}$ is a $(p, 2, s)$ atom. The "norm" $\|f\|_{H_{a}^{p, s}\left(\mathbb{R}^{n}\right)}$ is the infimum of all expressions $\left(\sum\left|c_{k}\right|^{p}\right)^{1 / p}$ for which we have a representation (2) of $f$. A well-known fact (see [FoS]) is that $\|f\|_{H_{a}^{p, s}\left(\mathbb{R}^{n}\right)} \cong\|f\|_{H^{p}\left(\mathbb{R}^{n}\right)}$, and in particular, $\|a\|_{H^{p}\left(\mathbf{R}^{n}\right)} \leq C$, with a constant $C$ independent of the $(p, 2, s)$ atom $a(x)$ if $s \geq[n(1 / p-1)]$.

We also have a similar decomposition theorem for any function $g \in H^{p}\left(\mathbb{T}^{n}\right)$. In particular, suppose $g \in H^{p}\left(\mathbb{T}^{n}\right) \cap \mathscr{S}\left(\mathbb{T}^{n}\right)$ and its Fourier coefficient

$$
a_{0}(g)=\int_{Q} g(x) d x=0
$$


where $Q=\left\{x \in \mathbb{R}^{n}:-1 / 2 \leq x_{j}<1 / 2, j=1,2, \ldots, n\right\}$ is the fundamental cube on which

$$
\int_{\mathbb{T}^{n}} g(x) d x=\int_{Q} g(x) d x
$$

for all functions $g$ on $\mathbb{T}^{n}$. Then we have the following lemma.

Lemma 4. Suppose $g \in H^{p}\left(\mathbb{T}^{n}\right) \cap \mathscr{S}\left(\mathbb{T}^{n}\right)$ with $a_{0}(g)=0$. If we restrict $x$ to $Q$, then for any fixed positive integer $s$

$$
g(x)=\sum c_{k} a_{k}(x)
$$

where each $a_{k}(x)$ is a $(p, 2, s)$ atom satisfying $a_{k}(x+n)=a_{k}(x)$ for $n \in \Lambda$ and $\|g\|_{H^{p}\left(\mathbb{T}^{n}\right)}^{p} \cong \sum\left|c_{k}\right|^{p}$.

Proof. Choose a radial function $\varphi \in \mathscr{S}\left(\mathbb{R}^{n}\right)$ with $\operatorname{supp}(\varphi) \subset B(0,1)$. In addition, we can choose such a $\varphi$ such that $\int x^{J} \varphi(x) d x=0$ for all multiindices $J,|J| \leq s$, and $\int_{0}^{\infty} \hat{\varphi}(t x)^{2} t^{-1} d t=1$ for all $x \neq 0$. We let $\tilde{\varphi}_{t}(x)=$ $\sum_{k \in \Lambda \backslash\{0\}} \hat{\varphi}(t k) e^{2 \pi i k \cdot x}=C \sum_{k \in \Lambda} t^{-n} \varphi((x+k) / t)$. Then by checking the Fourier coefficients, we easily obtain the following Calderón reproducing formula:

$$
g(x)=\int_{0}^{\infty}\left(\tilde{\varphi}_{t} * \tilde{\varphi}_{t} * g\right)(x) t^{-1} d t=\int_{0}^{1}+\int_{1}^{\infty} .
$$

Now by a standard argument $[\mathrm{FoS}]$ (or see $[\mathrm{BF}]$ for the proof on any compact Lie group), one can easily obtain that

$$
g(x)=\sum c_{k} a_{k}(x)
$$

where each $a_{k}(x)$ is a $(p, 2, s)$ atom satisfying $a_{k}(x+n)=a_{k}(x)$ for $n \in \Lambda$ and $\sum\left|c_{k}\right|^{p} \cong\left\|S_{\varphi}(g)\right\|_{L^{p}\left(\mathbb{T}^{n}\right)}^{p}$. Here $S_{\varphi}(g)$ is defined by

$$
S_{\varphi}(g)(x)=\int_{|x-y|<t}\left|\left(g * \tilde{\varphi}_{t}\right)(y)\right|^{2} t^{-n-1} d y d t .
$$

So to prove the lemma it suffices to show that $\left\|S_{\varphi}(g)\right\|_{L^{p}\left(\mathbb{T}^{n}\right)} \cong\|g\|_{H^{p}\left(\mathbb{T}^{n}\right)}$ for all $g \in H^{p}\left(\mathbb{T}^{n}\right) \cap \mathscr{S}\left(\mathbb{T}^{n}\right)$. But the proof for $\left\|S_{\varphi}(g)\right\|_{L^{p}\left(\mathbb{T}^{n}\right)} \cong\|g\|_{H^{p}\left(\mathbb{T}^{n}\right)}$ is, mutatis mutandis, the same as for $\mathbb{R}^{n}$ (see $[\mathrm{FoS}]$ ) without using any new techniques or ideas.

The following lemma is Lemma 3.1 in [F]. For completeness, we state its proof.

Lemma 6. Suppose that $\Psi(x)$ is a continuous function with compact support. Let $\lambda(x)$ be a bounded and continuous function on $\mathbb{R}^{n}$, and let $T_{\varepsilon}$ and $\widetilde{T}_{\varepsilon}$ be the families of operators on $\mathbb{R}^{n}$ and $\mathbb{T}^{n}$, respectively, associated to the function $\lambda$. Take $\Psi^{1 / N}(\xi)=\Psi(\xi / N)$. If $\Psi$ satisfies $\Psi(0)=1$ and $\hat{\Psi} \in L^{1}\left(\mathbb{R}^{n}\right)$, then for any $g \in \mathscr{S}\left(\mathbb{T}^{n}\right)$ and any positive integer $N$,

$$
\Psi(y / N)\left(\widetilde{T}_{\varepsilon} g\right)(y)=T_{\varepsilon}\left(g \Psi^{1 / N}\right)(y)+J_{N, \varepsilon}(y)
$$

for all $y \in \mathbb{R}^{n}$, where $J_{N, \varepsilon}(y)$ tends to zero uniformly for $y \in \mathbb{R}^{n}$ and $0 \leq \varepsilon \leq R$ $(R>0$ is any fixed number), as $N \rightarrow \infty$.

Proof. Since $g(x)=\sum a_{k}(g) e^{2 \pi i k \cdot x}$ with the Fourier coefficients $\left\{a_{k}(g)\right\}$ rapidly decreasing as $|k| \rightarrow \infty$, it suffices to prove the lemma when $g(x)=$ 
$e_{k}(x)=e^{2 \pi i k \cdot x}$. In this case

$$
\begin{aligned}
\left|J_{N, \varepsilon}(y)\right| & =\left|\Psi^{1 / N}(y)\left(\widetilde{T}_{\varepsilon} e_{k}\right)(y)-T_{\varepsilon}\left(\Psi^{1 / N} e_{k}\right)(y)\right| \\
& =\left|e_{k}(y) \int_{\mathbb{R}^{n}} N^{n} \hat{\Psi}(N \xi) e^{2 \pi i y \cdot \xi}\{\lambda(\varepsilon k)-\lambda(\varepsilon k+\varepsilon \xi)\} d \xi\right| \\
& \leq \int_{\mathbb{R}^{n}}|\widehat{\Psi}(\xi)|\left|\lambda(\varepsilon k)-\lambda\left(\varepsilon k+N^{-1} \varepsilon \xi\right)\right| d \xi .
\end{aligned}
$$

Since $\hat{\psi}$ is integrable and since $\lambda$ is bounded and continuous, the last quantity converges to zero as $N \rightarrow \infty$. The lemma is proved.

\section{PROOF OF THE MAIN THEOREM}

By a note on page 128 in [LL] we only need to show that for any $g \in$ $\mathscr{S}\left(\mathbb{T}^{n}\right) \cap H^{p}\left(\mathbb{T}^{n}\right)$ with $a_{0}(g)=0$,

$$
\left\|\tilde{T}^{*} g\right\|_{L^{p}\left(\mathbb{T}^{n}\right)} \leq C\|g\|_{H^{p}\left(\mathbb{T}^{n}\right)}
$$

For any $R>0$ fixed, we define $\widetilde{T}_{R} g(x)=\sup _{0<\varepsilon \leq R}\left|\widetilde{T}_{\varepsilon} g(x)\right|$. Since as $R \rightarrow \infty$ $\widetilde{T}_{R} g(x)$ increases pointwise to $\tilde{T}^{*} g(x)$, by monotonic convergence theorem, to prove $(8)$ we only need to prove that

$$
\left\|\widetilde{T}_{R} g\right\|_{L^{p}\left(\mathbb{T}^{n}\right)} \leq C\|g\|_{H^{p}\left(\mathbb{T}^{n}\right)}
$$

with a constant $C$ independent of $R$ and $g(x)$.

By Lemma $4, g(x)=\sum c_{k} a_{k}(x)$, where each $a_{k}$ is a $[n(1 / p-1)]+2 n$ atom and $\sum\left|c_{k}\right|^{p} \cong\|g\|_{H^{p}\left(\mathbb{T}^{n}\right)}^{p}$.

We let

$$
\Psi(x)=\prod_{j=1}^{n}\left(1-4 x_{j}^{2}\right)_{+}
$$

where

$$
f_{+}(x)= \begin{cases}f(x) & \text { if } f(x) \geq 0, \\ 0 & \text { if } f(x)<0 .\end{cases}
$$

For positive integers $M$ and $N$, we denote the cube $[-N / 2 M, N / 2 M)^{n}$ by $N Q / M$. Noting that $\widetilde{T}_{R} g(x)$ is a periodic function, for large $N$ we have

$$
\left\|\widetilde{T}_{R} g\right\|_{L^{p}\left(\mathbb{T}^{n}\right)}^{p} \cong N^{-n} \int_{N Q / 2}\left|\widetilde{T}_{R} g(x)\right|^{p} d x .
$$

Since on $N Q / 2$, there exists a constant $C>0$ such that $\Psi(x / N) \geq C$, it is easy to see that

$$
\left\|\widetilde{T}_{R} g\right\|_{L^{p}\left(\mathbb{T}^{n}\right)}^{p} \cong N^{-n} \int_{N Q / 2}\left|\Psi(x / N) \widetilde{T}_{R} g(x)\right|^{p} d x .
$$

By Lemma 6 and the assumption of the theorem, we have

$$
\begin{aligned}
\left\|\widetilde{T}_{R} g\right\|_{L^{p}\left(\mathbb{T}^{n}\right)}^{p \leq} & C N^{-n} \int_{\mathbb{R}^{n}}\left|T^{*}\left(g \Psi^{1 / N}\right)(x)\right|^{p} d x \\
& +c N^{-n} \int_{N Q / 2} \sup _{0<\varepsilon \leq R}\left|J_{N, \varepsilon}(x)\right|^{p} d x \\
\leq & C N^{-n}\left\|g \Psi^{1 / N}\right\|_{H^{p}\left(\mathbb{R}^{n}\right)}^{p}+o(1), \quad \text { as } N \rightarrow \infty .
\end{aligned}
$$


Now it suffices to show that for odd $N$

$$
\liminf _{N \rightarrow \infty} N^{-n}\left\|g \Psi^{1 / N}\right\|_{H^{p}\left(\mathbb{R}^{n}\right)}^{p} \leq C\|g\|_{H^{p}\left(\mathbb{T}^{n}\right)}^{p} .
$$

By Lemma 4 , we only need to prove that for ány $(p, 2, s)$ periodic atom $a(x)$ with support in $B\left(x_{0}, \rho\right) \subset Q$,

$$
N^{-n}\left\|a \Psi^{1 / N}\right\|_{H^{p}\left(\mathbb{R}^{n}\right)}^{p} \leq C,
$$

where $C$ is a constant independent of $a(x)$ and $N$.

By the definition, we have

$$
\begin{aligned}
& N^{-n}\left\|a \Psi^{1 / N}\right\|_{H^{p}\left(\mathbb{R}^{n}\right)}^{p} \cong N^{-n} \int_{\mathbb{R}^{n}} \sup _{0<t<\infty}\left|\int_{\mathbb{R}^{n}} \Psi(x / N) a(x) \Phi_{t}(y-x) d x\right|^{p} d y \\
& =N^{-n} \int_{\mathbb{R}^{n}} \sup _{0<t<\infty}\left|\int_{\mathbb{R}^{n}} \prod_{j=1}^{n}\left(1-4 x_{j}^{2} / N^{2}\right)_{+} a(x) \Phi_{t}(y-x) d x\right|^{p} d y \\
& =N^{-n} \int_{\mathbb{R}^{n}} \sup _{0<t<\infty}\left|\int_{\left|x_{j}\right|<N / 2}\left\{\prod_{j=1}^{n}\left(1-4 x_{j}^{2} / N^{2}\right) a(x)\right\} \Phi_{t}(y-x) d x\right|^{p} d y .
\end{aligned}
$$

Now we write $N=2 m+1$. Then, up to a set of measure 0 , the set $\{x \in$ $\left.\mathbb{R}^{n}:\left|x_{j}\right|<m+1 / 2, j=1,2, \ldots, n\right\}$ is the union of the disjoint sets $\{Q+$ $\left.k: k=\left(k_{1}, \ldots, k_{n}\right),-m \leq k_{j} \leq m, j=1,2, \ldots, n\right\}=\left\{Q_{k}\right\}$, where the $k_{j}$ 's are integers. Now the last integral above is bounded by

$$
I_{m}=C m^{-n} \sum_{-m \leq k_{j} \leq m} \int_{\mathbb{R}^{n}} \sup _{0<t<\infty}\left|\int_{Q_{k}}\left\{\prod_{j=1}^{n}\left(1-4 x_{j}^{2} / N^{2}\right) a(x)\right\} \Phi_{t}(y-x) d x\right|^{p} d y .
$$

Noting that $a(x)$ is a periodic function, we easily see that $\chi_{Q_{k}}(x) a(x)$ is an atom with support in $Q_{k}$, where $\chi_{Q_{k}}$ is the characteristic function of $Q_{k}$. Also since on $Q_{k}, \prod_{j=1}^{n}\left(1-4 x_{j}^{2} / N^{2}\right)$ is a polynomial of degree $2 n$ which is bounded by 1 , clearly

$$
\alpha(x)=\prod_{j=1}^{n}\left(1-4 x_{j}^{2} / N^{2}\right) \chi_{Q_{k}}(x) a(x)
$$

is a $(p, 2,[n(1 / p-1)])$ atom on $\mathbb{R}^{n}$. So the above integral $I_{m}$ is bounded by

$$
C m^{-n} \sum_{-m \leq k_{j} \leq m}\|\alpha\|_{H^{p}\left(\mathbb{R}^{n}\right)} \leq C .
$$

Theorem 2 is proved.

Following the proof on page 133 in [LL], we now easily obtain an improvement of Theorem 2 in [LL].

Theorem 3. Let $0<p \leq 1$, and let $1 \leq d<n$ be an integer. Suppose that $\lambda$ is a bounded and continuous function on $\mathbb{R}^{n}$. If $\lambda$ is a maximal multiplier on $H^{p}\left(\mathbb{R}^{n}\right) \quad\left(H^{p}\left(\mathbb{T}^{n}\right)\right)$, then the restriction of $\lambda$ to $\mathbb{R}^{d}$ is a maximal multiplier on $H^{p}\left(\mathbb{R}^{d}\right)\left(H^{p}\left(\mathbb{T}^{d}\right)\right)$.

\section{ACKNOWLEDGMENT}

We thank the referee for his helpful comments. 


\section{REFERENCES}

[BF] B. Blank and D. Fan, S-function, $g_{\lambda}$-functions and the Riesz potentials (submitted).

[F] D. Fan, Multipliers on certain function spaces, Rend. Circ. Mat. Palermo (2) 43 (1994).

[FS] C. Fefferman and E. M. Stein, $H^{p}$ space of several variables, Acta Math. (1972), 137-193.

[FoS] G. Folland and E. M. Stein, Hardy spaces on homogeneous groups, Princeton Univ. Press, Princeton, NJ, 1982.

[KT] C. Kenig and P. Tomas, Maximal operators defined by Fourier multiplier, Studia Math. 68 (1980), 79-83.

[LL] Z. Liu and S. Lu, Transference and restriction of maximal multiplier operators on Hardy spaces, Studia Math. 105 (1993), 121-134.

Department of Mathematical Sciences, University of Wisconsin-Milwaukee, MilwauKEE, WISCONSIN 53201

E-mail address: fan@csd4.csd.urm.edu

Department of Mathematics, University of Alabama, Tuscaloosa, Alabama 35487

E-mail address: ZWUQMATHDEPT. AS.UA.EDU 